\title{
Parametric design and static behaviour analysis of single-layer conical shell
}

\author{
Xiaoyang $\mathrm{Lu}^{1, \mathrm{a}}$, Yaru Wang ${ }^{2, \mathrm{~b}}$, Xiaoxiao Wang ${ }^{1, \mathrm{~b}}$, Hao Zhang $^{2, \mathrm{~b}}$, Silu $\mathrm{Xie}^{2, \mathrm{~b}}$ \\ ${ }^{1}$ Research Institute of Engineering Mechanics, Shandong Jianzhu University, Jinan 250101, China \\ ${ }^{2}$ Civil Engineering School, Shandong Jianzhu University, Jinan 250101, China \\ aluxy5504@163.com, b379982411@qq.com
}

Key words: single-layer conical shell; parametric design; comparison of static behaviou

Abstract: The parametric design macro programs of two basic types of single-layer conical shell are developed by using APDL(Ansys Parametric Design Language) parameterized design language.The parametric design of single-layer conical shell structures can be built under the condition of giving the macroscropic geometric parameters such as specifical span $S$, rise $f$, valley number $K n$, radial node number $N x$ etc.Ansys software is applied to study comparison of characteristics of the structure of different vector height $f$ and boundary constraintson. The results show that modeling design of conical shell can be built conveniently and quickly by the application of parametric design. The three-way grid is more reasonable force performance than Kiewitt.Thus it is suggested to give priority in the project.

\section{Introduction}

Reticulated has been proposed higher requirements on structural forms, with the rapid development of it at home and abroad and the continuous improvement of people's aesthetic standards[1].Many scholars at home and abroad have conducted more indepth research and discussion of spherical shells and cylindrical shells etc there common reticulated shell structures[2,3,4].However,for the conical reticulated shell,Xinzhi Wang[3] studied the nonlinear dynamic analysis of an equilateral triangular three-dimensional grid-type flat-cone single-layer lattice shell. The current research on this type of reticulated shell is not systematic enough and comprehensive.

In this paper, the parametric design language APDL[5] was used to develop two kinds of parametric design macros for single-layer conical mesh shells, which improved the efficiency of structural modeling and force analysis.Ansys software was used to analyze the mechanical behavior of structure with vector heights and boundary constraints.

\section{Geometric Descriptions of Structures}

According to the types of spherical shell, the conical shell is divided into five types: ribbed,Schwedler,Lamella,Kiewitt and three-way grid.The macroscopic geometric parametersits[1,6] are the span $S$, the vector height $f$, the number of circular region $K n$ and the number of radial nodes $N x$. Then taking the Kiewitt for example to describe the method of parametric design ( Figure 1).

\section{Parametric design of single-layer conical shell}

Taking Kiewitt (Fig.1) for example to describe parametric design of single-layer conical mesh shell due to space limitations. ( $i$ and $j$ are continuous positive integer).

1) Calculate the node coordinates and define the node number:In Cartesian coordinate system,the central axis of the cone is defined $Z$ axis, and the surface is divided into $K n$ sectors. Then define the 
most vertex node is No.1. Its coordinates are $(0,0, f)$.Dividing the radial length of the tapered bus bar to determine the $\mathrm{z}$ coordinate.Calculating the $x$ and $y$ coordinates of each node.Then the APDL loop statement[7] is used to number the nodes of circle $i$ and number $j$.The number is $1+K n \times(i-1) \times \mathrm{i} / 2+j$, $(i=1 \sim N x, j=1 \sim K n)$

2) Connect related nodes to form rods:Connecting Loop unit of the $i$-th circle firstly. 1 to $K n \times(i-1)$ symmetry area node number are $1+K n \times(i+1) \times i / 2+j$ and $1+K n \times(i+1) \times i / 2+j+1 . K n \times i$ symmetry area connection node number are $1+K n \times(i-1) \times i / 2+1$ and $1+K n \times(i-1) \times i / 2+K n \times i,(i=1 \sim N x, j=1 \sim K n \times i-1)$.

Connecting Radial Lever secondly.Connection node 1 and $1+j,(j=1 \sim K n) .1$ to $K n-1$ symmetry area node number are $1+K n \times(j-1) \times j / 2+(k-1) \times j+i$ and $1+K n \times(j+1) \times j / 2+(k-1) \times(j+1)+i . K n$ symmetry area node number are $1+K n \times(j-1) \times j / 2+1$ and $1+K n \times(j+1) \times j / 2+(k-1) \times(j+1)+i$. Forward link connection node number are $1+K n \times(j-1) \times j / 2+(k-1) \times j+i$ and $1+K n \times(j+1) \times j / 2+(k-1) \times(j+1)+i$. Negative link connection node number are $1+K n \times(j-1) \times j / 2+(k-1) \times j+i$ and $1+K n \times(j+1) \times j / 2+(k-1) \times(j+1)+i+1$ $(j=1 \sim N x-1, k=1 \sim K n, i=1 \sim j+1)$.

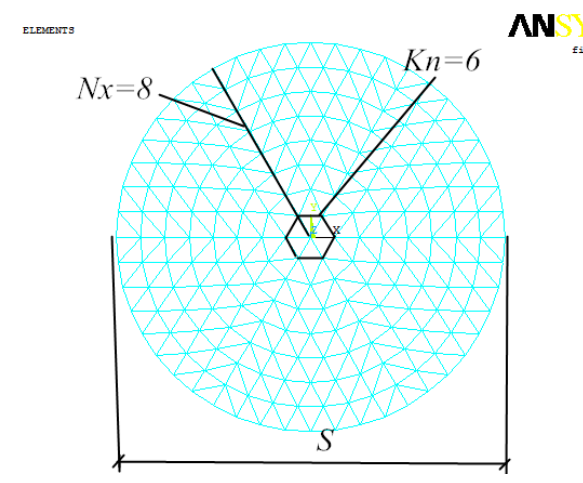

( a ) plan view

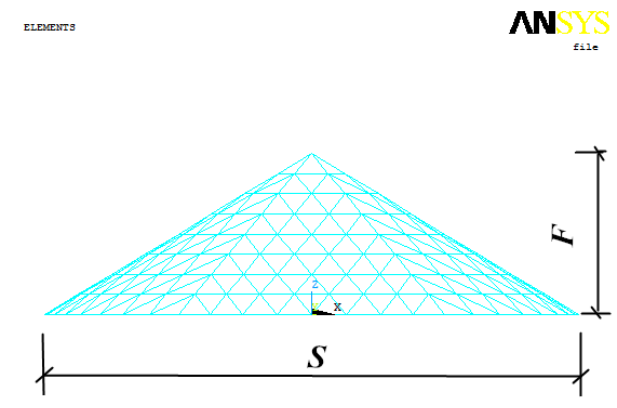

( b ) vertical view

Fig.1 Geometric Diagram

\section{Structural force performance comparison analysis}

The single-layer reticulated shell members are subject to greater axial forces,non-negligible bending moments and shear forces.So using hollow ball just nodes and space beam element.In this article,

Beam4 is used for the structural members, and Q235 seamless circular steel pipe $(\Phi 299 \times 10)$ is used for the structural members of the shell structure. The steel density is $p=7850 \mathrm{~kg} / \mathrm{m}^{3}$, the elastic modulus is $\mathrm{E}=2.06 \times 10^{11} \mathrm{MPa}$ and the Poisson's ratio $v=0.3$. The uniform cloth load of the roof is $2.35 \mathrm{kN} / \mathrm{m}^{2}[7]$ and comparative analysis of its mechanical properties. The allowable displacement of the structure is $1 / 400[8]$ of the span, and the allowable stress $[\sigma]=215 \mathrm{MPa}$. The ideal elasto-plastic material model is used.The material's strength and yield strength are not considered.

\section{Force performance}

Selecting the span $S=50 \mathrm{~m}$, the vector height $f=15 \mathrm{~m}$, the number of circumferential regions $K n=6$, and the number of radial node turns $N x=8$. The stress performance analysis is performed on the Kiewitt type and three-way grid type structures. The relevant data is shown in Table 1.

From Table 1,we know:The maximum displacement of two structures are far less than the allowable displacement of $125 \mathrm{~mm}$, so they meet the stiffness requirements. The most unfavorable stress is also 
far less than the allowable stress of $215 \mathrm{Mpa}$,so they meet the strength requirements. The structure is mainly subjected to compressive stress. The maximum displacement and unfavorable stress of the three-way grid type are small,and it can be seen that the stress performance of three-way grid is better than Kiewitt.

Table 1 Maximum Displacement and Maximum Stress of Two Types of Cone Reticulated Shells

\begin{tabular}{ccccc}
\hline Shell type & $\begin{array}{c}\text { Maximum } \\
\text { displacement }(\mathrm{cm})\end{array}$ & $\begin{array}{c}\text { displacement } \\
(\mathrm{cm})\end{array}$ & $\begin{array}{c}\text { Maximum } \\
\text { stress ( Mpa })\end{array}$ & $\begin{array}{c}\text { Allowable } \\
\text { stress( Mpa })\end{array}$ \\
\hline Kiewitt & 0.75 & 12.5 & 44.1 & 215 \\
Three-way grid & 0.67 & & 33.1 & \\
\hline
\end{tabular}

\section{Effect of Macroscopic Geometric Parameters on Mechanical Behavior of Structures}

The factors affecting the static performance of the reticulated shell include span, vector height, number of radial nodes, boundary constraints and so on. In this paper, the influences of vector height and boundary constraints on structural stress and displacement are studied.

\section{The influence of vector height on the mechanical properties of conical reticulated shells}

Select: $S=50 \mathrm{~m}, f=8 \mathrm{~m}, 10 \mathrm{~m}, 15 \mathrm{~m}, 20 \mathrm{~m}, 25 \mathrm{~m}, 30 \mathrm{~m}, 35 \mathrm{~m}, N x=8, K n=6$.Investigating the effect of vector height variation on structural stress and displacement. They are shown in Table 2.

Table 2 the most unfavorable stress and maximum displacement of Structure

\begin{tabular}{ccccccccc}
\hline Shell type & vector height $f$ & 8 & 10 & 15 & 20 & 25 & 30 & 35 \\
\hline Kiewitt & Maximum stress & 66.8 & 57.0 & 44.1 & 37.7 & 34.1 & 32.0 & 30.8 \\
Three-way grid & (Mpa) & 48.8 & 41.2 & 33.1 & 28.7 & 26.2 & 26.2 & 26.7 \\
Kiewitt & Maximum & 1.79 & 1.28 & 0.75 & 0.55 & 0.46 & 0.42 & 0.41 \\
Three-way grid & displacement (cm) & 1.62 & 1.15 & 0.67 & 0.51 & 0.44 & 0.40 & 0.41 \\
\hline
\end{tabular}

It can be seen from Table 2:The maximum stress and displacement of the three-way grid show a trend of decreasing with the increase of vector height. The most unfavorable stress and displacement have small difference with increasing vector height.So the influence on the strength and stiffness of the height change of the structure is close relatively.The maximum stress of Kiewitt and three-way grid are $37.2 \%$ and $27.1 \%$ of allowable stress. The maximum displacement of Kiewitt and three-way grid are $16.9 \%$ and $16.8 \%$ of the allowable displacement.So the stress is key factor to force performance.In the same conditions, the maximum stress and displacement of three-way grid is smaller than Kiewitt.Because three-way grid meshing more uniform than Kiewitt. So you can preferentially use three-way grid shell.

\section{Influence of bearing form reticulated shell structure}

Respectively using movable articulated joints and rigid joints (restricting all 6 degrees of freedom) to constrain two types of bearings, and other conditions are the same as before.Through static performance analysis, get the maximum stress and displacement are shown in Table 3.

We know that the two types are hinged or rigidly connected in two types of bearings, which have little effect on the maximum displacement of the structure, and have a significant impact on the most adverse structural stress. When the bearings are just attached, the most unfavorable stresses of the two types of structures increase, and the maximum displacement decreases. The most unfavorable stresses of the Kiewitt and the three-way increased by $21.77 \%$ and $81.27 \%$, 
respectively, and the maximum displacements decreased by $10.67 \%$ and $10.45 \%$ respectively. It can be seen that the change of the bearing type has the most obvious influence on the most unfavorable stress. In summary, the use of a hinged support is more advantageous to the structure.

Table 3 Effect of bearing form on structural static performance

\begin{tabular}{|c|c|c|c|c|c|}
\hline $\begin{array}{l}\text { structure } \\
\text { type }\end{array}$ & $\begin{array}{l}\text { Bearing } \\
\text { form }\end{array}$ & $\begin{array}{l}\text { Most adverse } \\
\text { stress ( } \mathrm{MPa} \text { ) }\end{array}$ & $\begin{array}{c}\text { Allowable } \\
\text { stress( MPa ) }\end{array}$ & $\begin{array}{c}\text { Maximum } \\
\text { displacement }(\mathrm{cm})\end{array}$ & $\begin{array}{c}\text { Allowable } \\
\text { displacement } \\
(\mathrm{cm})\end{array}$ \\
\hline \multirow{2}{*}{ Kiewitt } & Articulated & 44.1 & \multirow{4}{*}{215} & 0.75 & \multirow{4}{*}{12.5} \\
\hline & Just connected & 53.7 & & 0.67 & \\
\hline \multirow{2}{*}{$\begin{array}{c}\text { Three-wa } \\
\text { y grid }\end{array}$} & Articulated & 33.1 & & 0.67 & \\
\hline & Just connected & 60.0 & & 0.60 & \\
\hline
\end{tabular}

\section{Conclusion}

The APDL parameterized design language was used to develop two kinds of design programs for single-layer conical mesh shell structures. The influence of different geometrical parameters on the static performance of conical latticed shells was investigated. The results show that:

(1) The maximum stress and displacement of the two types of reticulated shells are decrease with the increase of vector height.

(2)The stress is the key factor to force performance of the conical reticulated shells.So the strength of the entire structure can be improved by increasing the strength of the corresponding rods.

(3)Since the three-way grid lattice shell is a regular triangular grid, the grid division is relatively uniform and the stress is reasonable. Therefore in practical engineering design, three-way grid type conical lattice shells can be considered preferentially.

\section{Acknowledgements}

This work was financially supported by projects of Shandong postgraduate innovation (SDYY08038) and postgraduate high quality courses of Shandong Jianzhu University (YZKC201605).

\section{References}

[1] Xiaoyang Lu,Xiaowei Zhao,Shiying Chen.Optimum Design of Discrete Variable Shell Structure[M].Beijing: China Architecture \& Building Press,2013.

[2] Yongjun He,Xianhong Cao.Static and Stability Analysis of Rotating Sinusoidal Surface Single Layer Reticulated Shell[J]. Building Science,2016,32(9):1-7

[3] Xinzhi Wang,Congxing Liang ,Xuexing Ding.Nonlinear Dynamic Stability Analysis of Shallow Cone Reticulated Shells[J]. Engineering Mechanics,2005,22.

[4] Yaruo Chen,Shaobo Lu,Xiaoyang Lu.The fusion of traditional architectural style and modern lattice shell structure[J].Journal of Shandong Jianzhu University,2014,29(1):33-39

[5] Shuguang Gong,Guilan Xie.ANSYS Parametric Programming and Command Manual.Beijing: Mechanical Industry Press, 2010

[6] Long Li,Shaobo Lu,Xiaoyang Lu.Analysis of Static and Anti-seismic Performance of Dashi High Rectangle Fork Cylindrical Shell[J].Science Technology and Engineering,2016,14:268-275. [7] GB50009-2012.Building structural load specification[S].Beijing:China Building Industry Press, 
2012.

[8] JGJ7-2010.Technical Specifications of Spatial Grid Structure[S].Beijing: China Building Industry Press, 2010. 